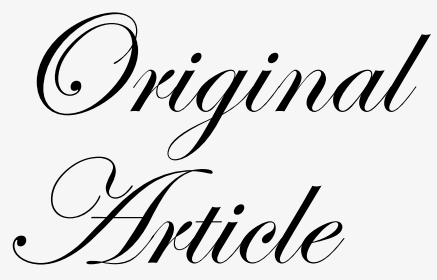

${ }^{1}$ Centre for Liver Research, School of Digestive \& Liver Diseases, Institute of Post Graduate Medical Education \& Research, Kolkata, India

${ }^{2}$ Indian Institute of Liver and Digestive Sciences, Kolkata, India

\#Co- Corresponding authors: Amal Santra, Ph.D, Professor, Centre for Liver Research, School of Digestive \& Liver Diseases, Institute of Post Graduate Medical Education \& Research, Kolkata 700020;India; email: asantra2000@yahoo.co.in

Abhijit Chowdhury, M.D., D.M., Professor of Hepatology, School of Digestive \& Liver Diseases, Institute of Post Graduate Medical Education \& Research, Kolkata 700020; India; email: achowdhury2002@ yahoo.co.in

\section{Induction of Heme oxygenase 1 protects hepatocytes from Isoniazid - Rifampicin induced cell death: an in vitro study}

\author{
Debasree Bishnu ${ }^{1, \#}$, Suman Santra ${ }^{1, \#}$, Gopal Krishna Dhali ${ }^{1}$, Abhijit \\ Chowdhury $^{1,2}$, Amal Santra ${ }^{1,2}$
}

\begin{abstract}
Background: Anti tuberculosis therapy agent isoniazid (INH) and rifampicin (RMP) injure hepatocytes. Heme oxygenase-1(HO-1) is a stress induced protein which seems to have some cellular protective function. We examined the protective function of HO-1 during INH-RMP induced cell death of hepatocytes by induction of HO-1 using hemin chloride or by silencing HO-1 gene using small interfering RNA (siRNA).

Methods: The role of HO- 1 induction on INH-RMP induced cell death was examined on HepG2 cells overexpressing human CYP2E1 gene (E47 cells) during short term culture. The E47 cells were treated with hemin chloride to induce HO-1 expression during INH-RMP treatment. In other set of experiments, transient knockdown of HO-1 gene using siRNA was carried out before treatment of INH-RMP. Cell viability using Trypan blue, intracellular reactive oxygen species (ROS), cell death were evaluated by FACS analysis at different time points of INH-RMP treatment.

Results: INH-RMP treatment to E47 cells induced expression of cytoplasmic HO-1 protein at early hours of drug treatment with minimum loss of cell viability and cell death. At later hours, failiure to express HO- 1 protein resulted in loss of cell viability and increased cell death. Addition of Hemin chloride during treatment of INH-RMP induced HO-1 in E47 cells and reversed the drug induced liver injury. Silencing the HO-1 gene using siRNA potentiated INH-RMP induced cell death of the E47 cells. Conclusion: Induction of HO-1 ameliorated INH-RMP induced cell death of hepatocytes. This may be a potential target for future therapeutic option in INHRMP induced drug induced liver injury.
\end{abstract}

KEYWORDS: Anti-tubercular drugs, heme oxygenase 1; hepatocyte injury 


\section{Introduction:}

Hepatotoxicity resulting from anti-tubercular drugs (ATD) is one of the commonest causes of drug induced liver injury (DILI) in India. ${ }^{1,2}$ Among the first line AT drugs, Isoniazid (INH) is the most potent hepatotoxin causing serious hepatotoxicity ranging from asymptomatic rise in serum transaminases to acute liver failure with a high mortality rate. ${ }^{3,4}$ Rifampicin (RMP) which generally used with INH as a first line agent to treat tuberculosis, potentiates INH induced liver injury. ${ }^{5}$ On the basis of different regimens and definitions of hepatic injury, the incidence of ATD induced hepatotoxicity is reported to occur in $1 \%$ to $36 \%{ }^{4}$

Since oxidative stress plays an important role in INHRMP induced toxicity, use of antioxidant enzymes and other antioxidants to protect against oxidative injury can be a fruitful strategy. Heme Oxygenase (HO), a microsomal enzyme, has diverse physiological functions in the liver. HO has three isoenzymes and among these isoenzymes, Heme Oxygenase-1 (HO-1) is a $32 \mathrm{kd}$ heat shock protein. ${ }^{6,7}$ Like other stress proteins, $\mathrm{HO}-1$ is induced by oxidative stress, low level of cellular GSH (reduced glutathione) level ${ }^{8}$ and has the potential to protect against oxidative injury and inhibit inflammation. ${ }^{9} \quad$ Further, HO-1 is also shown to exhibit anti apoptotic and anti proliferative properties. ${ }^{10}$ On the other hand, HO-2 and HO-3 are constitutively expressed in normal physiological conditions. ${ }^{9}$ Hence, elevated expression of HO- 1 serves as the most critical cellular defence protein during cellular stress by its anti-oxidative and anti apoptotic properties.

The primary goal of the current study was to evaluate whether HO-1 can offer protection against INH-RMP induced toxicity in CYP2E1 over expressing HepG2 cells (E47 cells). HO-1 expression in E47 cells was enhanced by challenging with hemin chloride, an inducer of HO-1 or decreased by siRNA HO-1 treatment. The consequence of modulating cellular HO-1 levels on INH-RMP induced cellular injury was determined.

\section{Materials and Methods}

\section{Cells and chemicals}

Human hepatoma HepG2 cell line that over express human CYP2E1 (E47 cells) was used in this study. ${ }^{11}$ E47 cells were kindly provided by AI Cederbaum (Mount
Sinai School of Medicine, New York, U.S.A.). E47 cells were cultured in minimum essential media (MEM) containing $10 \%$ fetal bovine serum and $0.5 \mathrm{mg} / \mathrm{ml}$ of G418 aminoglycoside supplemented with 100 units/ $\mathrm{ml}$ of penicillin and $100 \mu \mathrm{g} / \mathrm{ml}$ of streptomycin in a humidified atmosphere in presence of $5 \% \mathrm{CO}_{2}$ at $37^{\circ} \mathrm{C}$. Media, antibiotics and most of the reagents used for cell culture were purchased from Sigma Aldrich.

\section{INH-RMP treatment}

E47 cells ( $1 \mathrm{X} 10^{5}$ cells) as well as HepG2 (1X10 cells) cells were plated and incubated in growth medium (MEM) overnight prior to different treatments till 60\% confluency. They were treated with $5 \mu \mathrm{M}$ INH and 2.5 $\mu \mathrm{M}$ RMP for different time periods $(1-24$ hours). At the end of the pre-determined treatment schedule, cells were processed for cell viability assay using Trypan blue dye exclusion technique. Further intracellular ROS, cell death was assessed in each time point.

Induction of Heme oxygenase 1 in E47 cells

To evaluate the protective role of HO-1 in INH-RMP induced injury of hepatocytes, an additional experiment was carried out where cellular HO-1 was induced by addition of $20 \mu \mathrm{M}$ hemin chloride (hemin) in the culture medium $3 \mathrm{hr}$ before addition of INH-RMP to $1 \mathrm{X} 10^{5}$ E47 cells (HepG2 cells expressing CYP2E1) and 1 X10 $10^{5}$ HepG2 cells.

\section{Knockdown of Heme oxygenase 1 in $E 47$ cells}

To silence Heme oxygenase 1 (HO-1) expression in E47 cells, HO-1 siRNA for human (siRNA; sc-35554, purchased from Santa Cruz Biotechnology, Dallas, U.S.A.) was used. E47 cells $\left(1 \mathrm{X} 10^{5}\right)$ were cultured one day prior to transfection in 12-welled culture plates. Transfection of HO-1 siRNA or scrambled siRNA (sc-37007) was carried out using X-tremeGENE HP DNA Transfection Reagent (Roche Diagnostic, Germany) as per protocol of the manufacturer.After overnight transfection, cells were incubated with or without INH-RMP for different time periods before measuring intracellular ROS, cell death and other parameters. The efficiency of HO-1 knockdown was evaluated by western blot analysis. 
DCF staining and flow cytometry for detection of ROS

Intracellular ROS was assessed using fluorescent probe 2', 7'-dichlorofluorescin diacetate (DCF-DA) of the cultured E47 cells and HepG2 cells at different time points of INH-RMP treatment by flow cytometer (BD FACS Calibur, BD Bioscience, Pharmingen, U.S.A.) using FL1 channel to detect DCF fluorescence. ${ }^{12}$ In brief, 30 minutes before the end of treatment schedule in the presence or absence of INH-RMP, E47 cells and HepG2 cells were incubated with $5 \mu \mathrm{M}$ DCF-DA in MEM for $30 \mathrm{~min}$ at $37^{\circ} \mathrm{C}$ in the dark. The cells were washed in PBS, trypsinized, and resuspended in PBS. The intensity of fluorescence wasdetected from 10,000 cells by flow cytometry using Cell Quest software.

Annexin $V$ and Propidium iodide staining and flow cytometry for detection of apoptosis

After specified time point of INH-RMP treatment, methanol-acetic acid fixed cells were stained with Propidium iodide (PI) and Annexin V using AnnexinV-Fluos staining kit (Roche, Germany). Cells were collected after treatment with or without INH-RMP, washed twice in ice-cold PBS, and resuspended in binding buffer at a density of $1 \times 10^{5}$ cells $/ \mathrm{ml}$. The cells were stained with Fluorescein-labeled Annexin V and PI for $15 \mathrm{~min}$ as per protocol of the manufacturer. The intensity of fluorescence was detected from 10,000 cells by flow cytometry (BD FACS Calibur, BD Bioscience, Pharmingen, U.S.A.) using FL1 channel (for Fluoresceinlabeled Annexin V) and FL2 channel (for PI).

\section{Preparation of cell lysate}

Whole cell lysate was prepared using RIPA buffer (Cell Signaling Technology, U.S.A.) with protease inhibitor cocktail (Roche Diagnostics, Mannheim, Germany). The lysates were centrifuged at $14,000 \mathrm{xg}$ for 20 minutes at $4^{\circ} \mathrm{C}$. The protein content of the supernatant was determined with the Bradford protein assay kit (Sigma, U.S.A.) as recommended by the manufacturer.

Western blotting
Forty micrograms protein was resolved on SDS-PAGE after denaturation in sample buffer and transferred onto PVDF membranes (Thermo Fisher Scientific, U.S.A.). After blocking with 5\% skimmed milk, the blots were probed with the following antibodies: mouse monoclonal anti HO-1 and mouse monoclonal anti beta actin, (Santa Cruz Biotechnology). Horseradish peroxidase (HRP)conjugated goat anti-mouse IgG (1:1000) (Santa Cruz Biotechnology) was used as secondary antibody. Blots were developed using the enhanced chemiluminescence immunoblot-detecting reagent (Thermo Scientific, U.S.A.).

\section{Caspase 3 activity assay}

The activity of caspase 3 was determined in cells homogenates by measuring proteolytic cleavage of the specific fluorogenic substrates DEVD-AFC (Asp-GluVal-Asp) (AFC: 7-amino-4-trifluoromethyl coumarin, respectively; BioVision).

\section{Statistical Analysis}

All experiments were replicated at least five times. Data were presented as means \pm standard deviation (SD). The Student's $\mathrm{t}$ test was used to evaluate statistical differences between groups. A p value less than 0.05 was considered statistically significant.

\section{Results}

INH-RMP potentiates CYP2E1 induced intracellular ROS mediated cell death and expression of HO-1 in E47 cells:

Our previous study documented development of oxidative stress in the liver during INH-RMP treatment in mouse model. ${ }^{13}$ In this study, we first assessed the intracellular ROS in the E47 cell line during INH-RMP treatment using 2,7 dichlorofluorescein diacetate (DCFDA), a probe which is oxidized by several oxidants and high DCF fluorescence was an indicator of intracellular ROS generation. Using flow cytometric analysis (FACS), we determined the percentage of cells producing high DCF fluorescence level with or without INH-RMP treatment at different time points. Throughout 24 hours 
of culture of CYP2E1 expressing E47 cells without INHRMP treatment, high DCF fluorescence was detected in less than $10 \%$ of cells (Figure 1A) and cell viability remained $95 \%$ to $98 \%$ as assessed by trypan blue dye exclusion technique. In contrast, E47 cells treated with INH-RMP showed higher percentage of cells with high DCF fluorescence starting from first hour mark and this figure progressively increased with increasing duration of INH-RMP exposure (Figure 1A). At $6 \mathrm{hr}$ and 24 hour post treatment with INH-RMP $54 \%$ and $74 \%$ of E47 cells, respectively, had high DCF. As high DCF is an indicator of increased intracellular ROS generation, we assessed cell viability at the different time points of post INH-RMP treatment. Using trypan blue dye exclusion technique, $90 \%$ to $94 \%$ cells remained viable at $6 \mathrm{hr}$ post INH-RMP treatment which declined to $70 \%$ to $72 \%$ at $12 \mathrm{hr}$ and $56 \%$ to $62 \%$ at $24 \mathrm{hr}$ respectively. An interesting observation in this study was despite high DCF fluorescence in 54\% of E47 cells at $6 \mathrm{hr}$ of drug treatment, $90 \%$ to $94 \%$ cells remained viable. This may be due to some stress induced protective molecule in the hepatocytes expressed in response to the increased intracellular oxidative stress. On the other hand, in HepG2 cells, no change in intracellular ROS level and loss of cell viability was observed (data not shown).

Any agent that can cause oxidative stress may induce HO-1. HO-1 is a possible agent to initiate hepatoprotective and anti-inflammatory activity. ${ }^{14}$ We therefore checked whether HO-1 was induced in the INH-RMP treated E47 cells. At basal condition as well as in control experiments low level of immunoreactive HO-1 was observed. In presence of INH-RMP, immunoreactive HO-1 was progressively increased in E47 cells till $6 \mathrm{hr}$ and was gradually declined at 12 and 24 hrs of drug treatment as detected by Western blot technique (Figure 1B) whereas no significant changes were observed in expression of HO-1 in HepG2 cells after INH-RMP when compared to expression at $0 \mathrm{hr}$ (Figure 1B).

FACS analysis using propidium iodide and annexin $\mathrm{V}$ staining revealed that E47 cells treated with INHRMP exhibited mainly apoptotic death. Annexin V has high affinity to bind with phosphatidylserine (PS). PS is normally found on the intracellular leaflet of the plasma membrane in healthy cells. Externalization of the PS is a marker of apoptosis of the cells and externalization of PS can be detected using fluorochrome labeled Annexin $\mathrm{V}$ by flow cytometry. On the other hand, necrotic cells show bright red fluorescence of PI and no green fluorescence of Annexin V. Living cells show no green or red fluorescence. Till 6 hour of INH-RMP treatment the cell death was found to be low. However, rapid increase of apoptotic death was noted at 12 and $24 \mathrm{hrs}$ of INHRMP treatment (Figure 1C). Caspase activation is one of the characteristic features of apoptosis. The INH-RMP induced apoptotic death of the E47 cells was further confirmed by increased caspase 3 activity at 12 and 24 hrs (Figure 1D).

\section{HO-1 siRNA enhances increase of ROS by INH-RMP in E47 Cells}

To study whether HO-1 protects E47 cells from INH-RMP dependent oxidative stress, HO-1 siRNA and siRNAcontrol (a nontargeting siRNA) (Santa CruzBiotechnology) were transfected using the siRNA transfection reagent according to the manufacturer's protocol and then challenged with INH-RMP for different time periods. Since we observed maximum HO-1 induction at 3 and 6 hours in response to INH-RMP treatment, we carried out our experiments at 3 and 6 hours of INH-RMP treatment following knockdown of HO-1 mRNA. Knockdown of HO-1 mRNA caused rapid increase of E47 cells with high DCF fluorescence following INH-RMP treatment at 3 and 6 hours (Figure 2A).

HO-1 siRNA inhibits HO-1 protein expression in vitro To evaluate the role of HO-1 in the pathophysiology of INH-RMP induced toxicity, we first tried to knock down HO-1 induction by using an HO-1 siRNA approach. Knockdown of HO-1 mRNA using an HO-1 siRNA caused more than $60 \%$ reduction in HO- 1 protein in E47 cells at $6 \mathrm{hr}$ of INH-RMP treatment, whereas the nonspecific siRNA had no measurable effect on HO-1 expression (Figure 2B).

\section{Knockdown of HO-1 mRNA Potentiates the Toxicity Caused by INH-RMP in E47 Cells}

To determine the protective effect of HO- 1 on cell death, knockdown of HO-1 mRNA was carried out before INH- 
A.

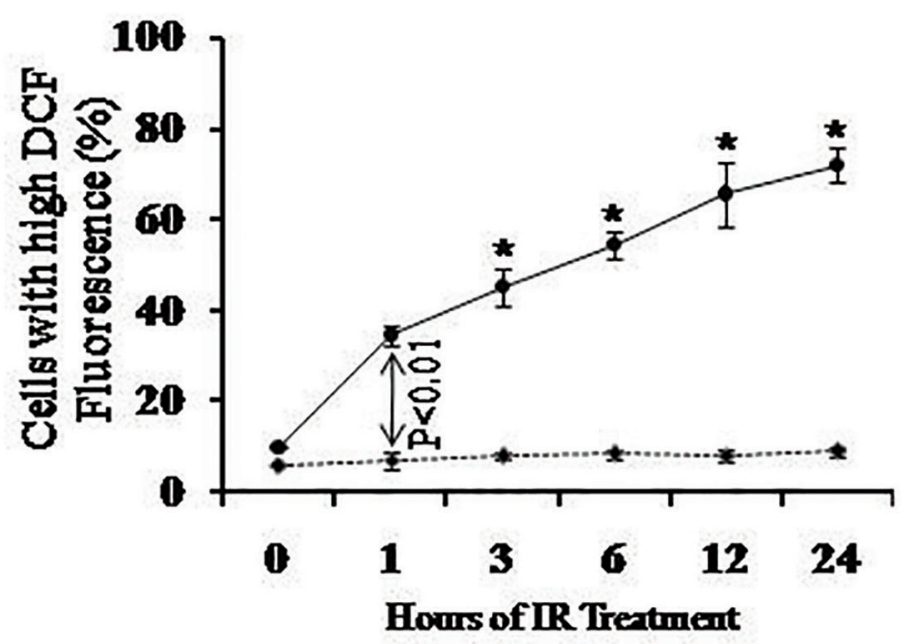

B.

\section{Hours of IR Treatment}

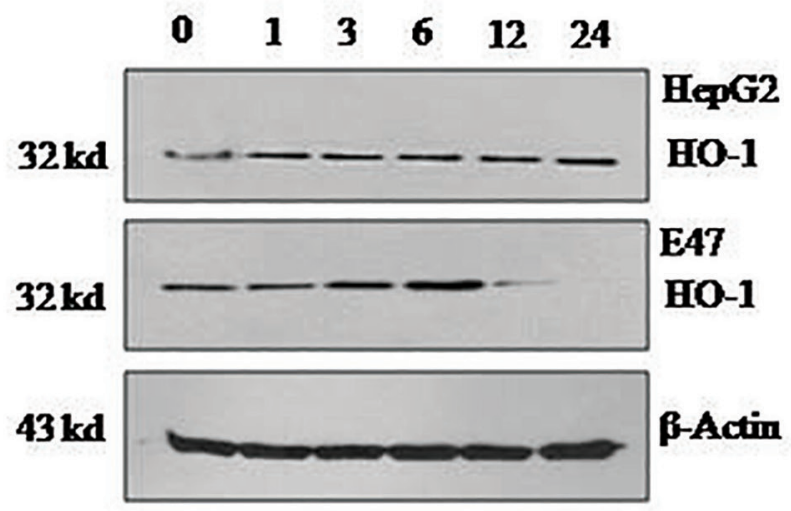

C.

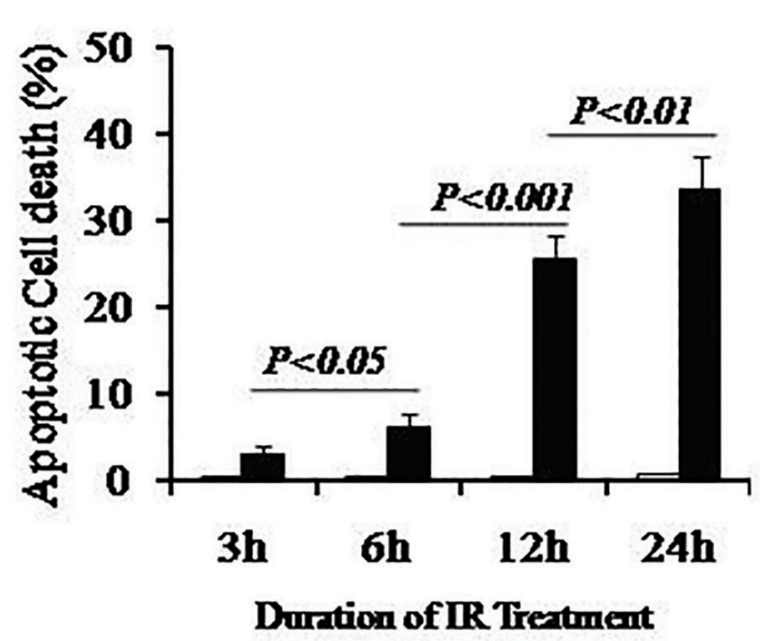

D.

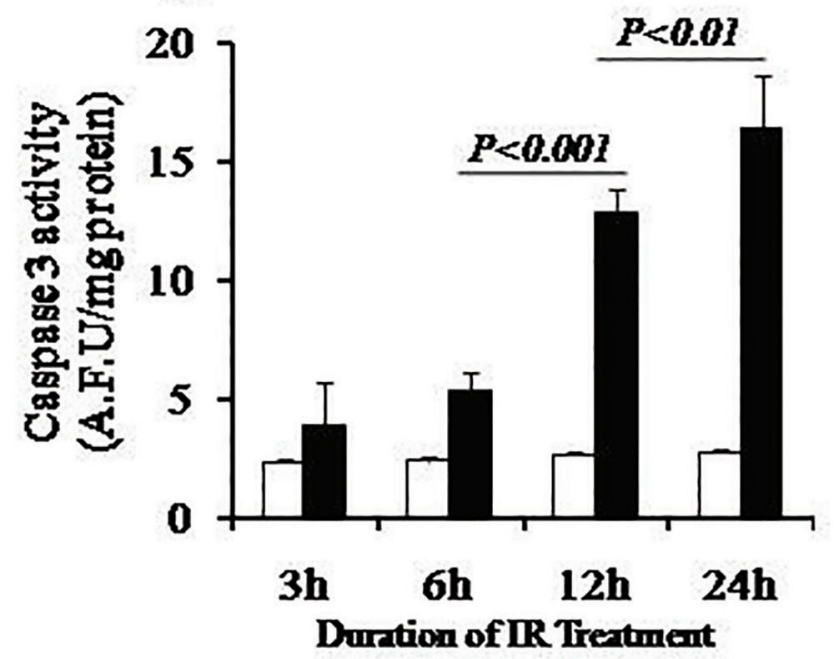

Figure 1: Intracellular ROS, induction of HO-1 and cell death in E47 cells exposed to isoniazid and rifampicin (IR) at different time points. A) E47 cells treated with IR produced increased intracellular ROS as detected by FACS analysis after staining with DCFDA. Solid line indicates E47 cells treated with IR while dotted line indicated cells without IR treatment. ${ }^{*} \mathrm{p}<0.05$ compared to the previous time point B) Western blot analysis revealed induction of HO-1 in E47 cells at 3 and 6 hours of IR treatment. Expression of HO- 1 gradually diminished at 12 and 24 hours of IR treatment whereas HO-1 expression remain unchanged in HepG2 cells following INH-RMP treatment at different time points C) Apoptotic death of the E47 cells after different hrs of IR treatment was shown in the bar diagram. Analysis of cell death was evaluated by FACS analysis after staining with Annexin V and PI staining. D) Increased cell death of the $\mathrm{E} 47$ cells due to IR treatment was confirmed by Caspase 3 activity as depicted here. Black bars indicate cells treated with IR, while white bars indicate cells without IR treatment. ${ }^{*} \mathrm{p}<0.05$ compared to cells without IR treatment. 
RMP treatment. HO-1 knockdown caused rapid increase in E47 cells undergoing apoptosis,measured using Annexin V and PI, at 3 hour and 6 hour post INH-RMP treatment (Figure 2C). To further confirm, caspase 3 activity assay was carried out in our experimental system. Blocking HO-1 mRNA by siRNA-HO-1, markedly increased caspase 3 activity compared with those in control siRNA group and INH-RMP treated group without knockdown of HO-1 mRNA (Figure 2D).

\section{Overexpression of HO-1 Confers E47 Cell Resistance to INH-RMP induced Toxicity}

Further, to confirm the protective effects of HO-1 against INH-RMP toxicity, E47 cells were pre-treated with hemin chloride $(20 \mu \mathrm{M})$, a classical inducer of $\mathrm{HO}-1^{15}$, to carry out the HO-1 over expression experiments. Pre-treatment with hemin chloride resulted in a 2 fold induction of HO-1 as demonstrated by western blots (Figure 3A).

E47 cells treated with INH-RMP had fewer cells producing significant ROS measured at both 3 and 6 hours in the group where HO-1 overexpression was induced using hemin chloride as compared to the group treated with INH-RMP but no induction with hemin (Figure 3B).

HO-1 over expression exhibited significant reduction of apoptosis of E47 cells caused by treatment with INHRMP (Figure 3C). These data was further confirmed by assessment of caspase 3 activity (Figure 3D). Further, induction of HO-1 in E47 cells with hemin chloride also elicited resistance to INH-RMP induced intracellular ROS generation and cell death till 24 hour at all the time points that we have studied.

\section{Discussion}

This is an in-vitro study searching for identification of a protective agent to prevent anti tubercular (AT) drug induced hepatocyte injury and death. AT drugs induced acute liver failure is the major cause of drug related acute liver failure in India. ${ }^{1,2}$ In this study we find that induction of HO-1 in hepatocytes ameliorates INH-RMP induced injury and cell death.

Since INH-RMP treatment induces oxidant-mediated liver cell injury, therapeutic use of antioxidative enzymes could be an attractive and unexplored treatment option. ${ }^{13}$ HO-1 is a heat shock cytoprotective protein having molecular weight $32 \mathrm{kd} .{ }^{6,7}$ It is induced in the cell in response to hypoxia, UV light and intracellular oxidative stress. ${ }^{16}$ This microsomal enzyme has anti-oxidative, anti-inflammatory and anti-apoptotic functions and thus prevents the cells against various toxic agents. ${ }^{17}$ However, the beneficial effects of HO-1 in AT drugs induced liver injury is still not clear. In this in-vitro study, we used E47HepG2 cells that constitutively express human CYP2E1 because CYP2E1, the major microsomal catalysing enzyme is involved in the oxidative metabolism of INH and production of hepatotoxic intermediaries. ${ }^{18} \mathrm{HO}-1$ induction was observed in the livers of chronic alcoholfed mice or pyrazole-treated rats, conditions known to elevate CYP2E1 levels. ${ }^{19}$ Compounds known to be actively metabolized by CYP2E1 to reactive intermediates such as acetaminophen or carbon tetrachloride were found by previous studies to be toxic to the E47 cells but not to the HepG2 cells, validating the use of E47 cells to study CYP2E1-dependent toxicity. ${ }^{20}$ We found by immunoblot analysis that HepG2 cells did not express CYP2E1 and treatment of these cells with INH-RMP for 24 hours did not affect its expression (data not shown). In contrast, a strong immunoreactive signal of basal CYP2E1 protein was detected in E47 cells (data not shown). Further the rationality of using E47 cells is justified because previous human studies have shown the involvement of CYP2E1 in INH induced hepatotoxicity ${ }^{21}$, but direct evidence is not available. Hepatic enzyme $\mathrm{N}$-acetyltransferase 2 acetylates INH to acetylisoniazid which is first hydrolysed to acetylhydrazine and subsequently oxidized by CYP2E1 to produce hepatotoxic intermediates. ${ }^{18}$ Metushi et al reported that INH is bioactivated by, and covalently binds to, CYP2E1, in the presence of a NADPH regenerating system $^{22}$ and the data of this study was found to be consistent with the observation that the high activity variant CYP2E1 c1/c1 genotype is associated with more severe liver injury. ${ }^{21}$

In this study, we found that the HO-1 is important in protecting E47 cells against INH-RMP induced toxicity. CYP2E1 over expression results in repression of HO-1 as evidenced by western blot during oxidative injury after 6 hours of INH-RMP treatment, resulting 
A.

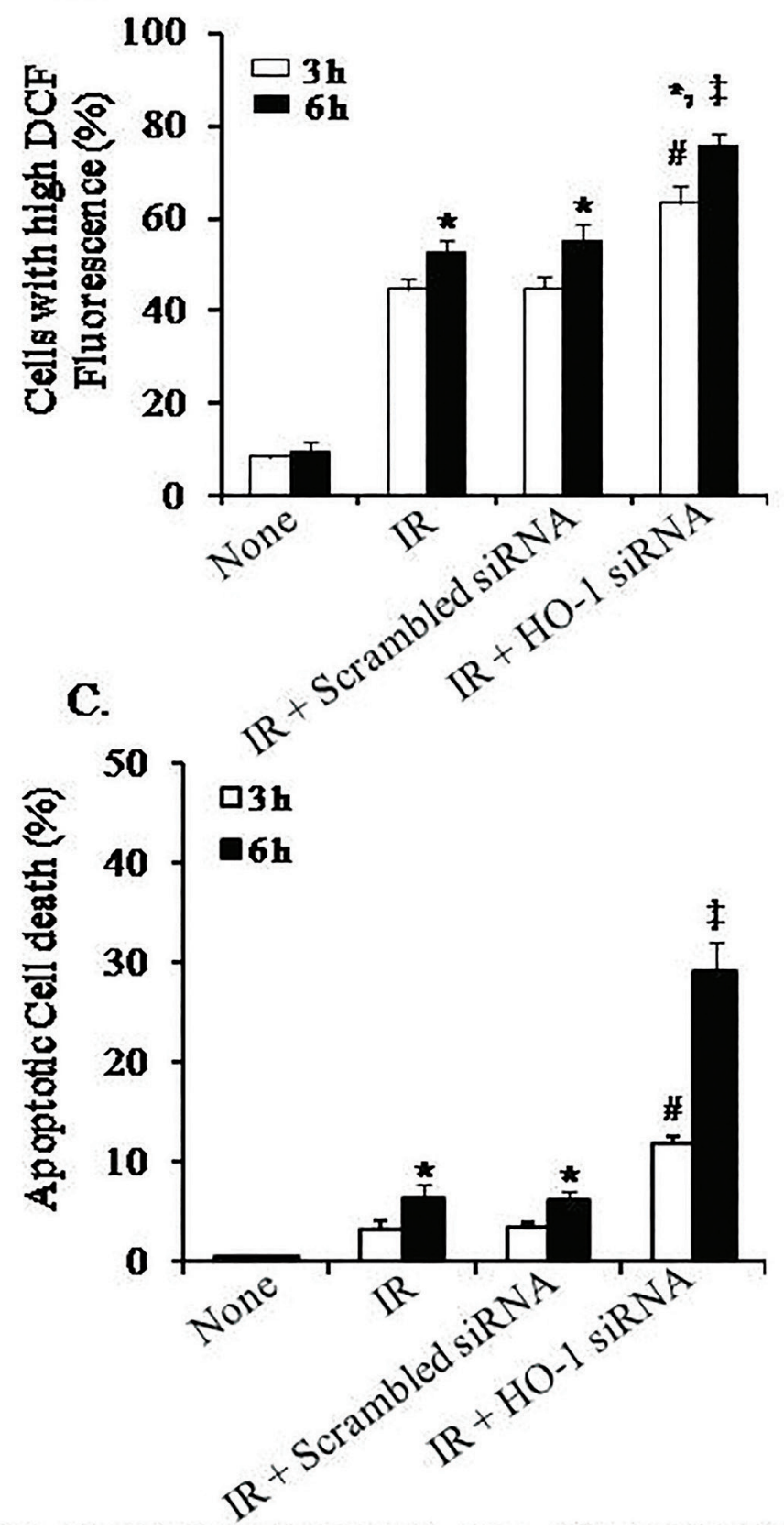

B.

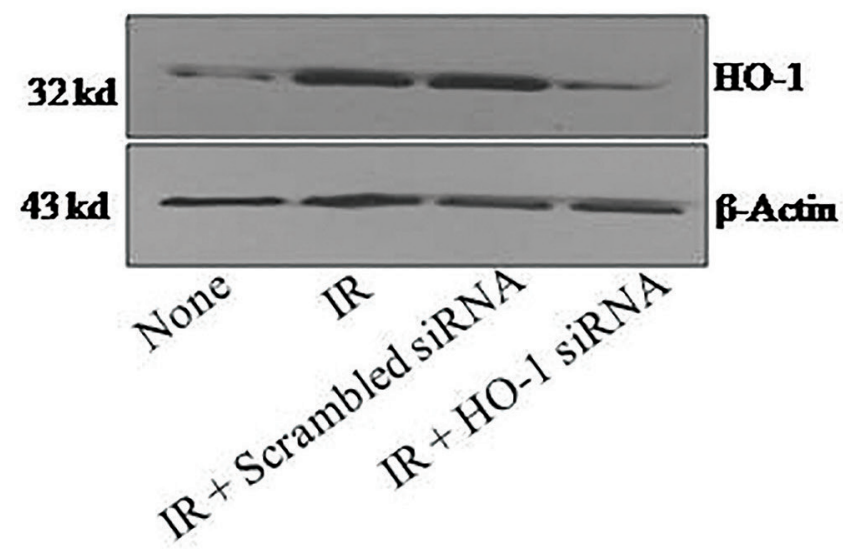

D.

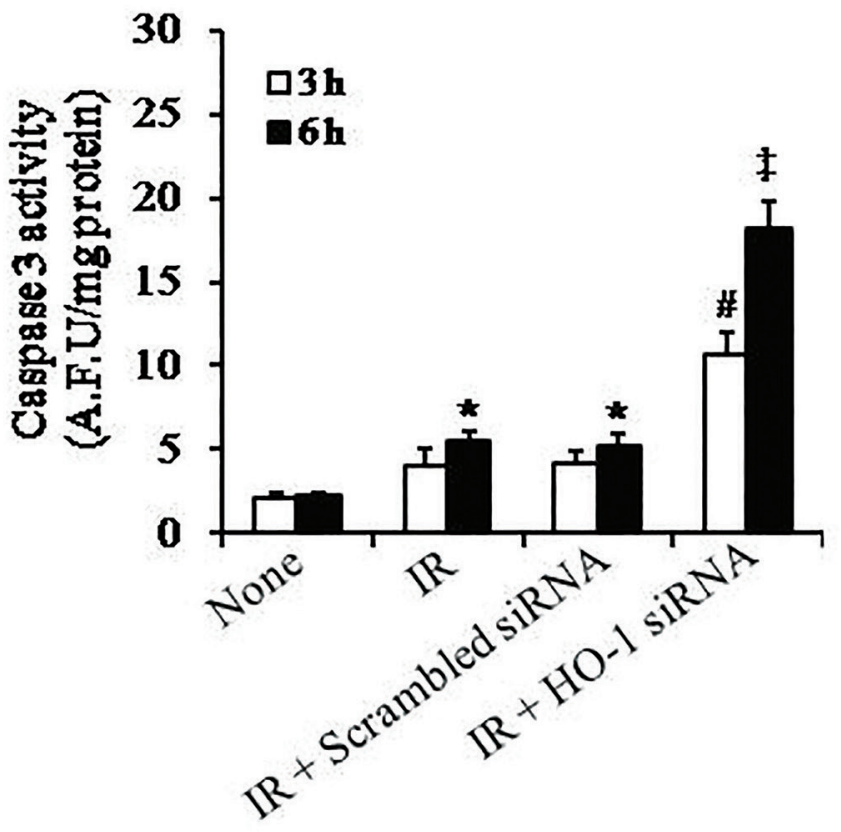

Figure 2: Effect of HO-1 knockdown on susceptibility to E47 cells to IR treatment. HO-1 knockdown was carried out by transfection of HO-1 siRNA into E47 cells. A) Knockdown of HO-1 mRNA results increased percentage of E47 cells producing high DCF fluorescence at 3 and 6 hrs of IR treatment. Black bars indicate IR treatment for 6 hr while white bars indicate IR treatment for $3 \mathrm{hr}$. B) Western blot analysis confirm knockdown of HO-1 in E47 cells as documented significant reduction of HO-1 protein in E47 cells at 6 hr of IR treatment. C) Silencing HO-1 mRNA in E47 cells results rapid increase of percentage of cell death due to IR treatment at 3 and 6 hrs. D) Rapid increase of caspase 3 activity in IR treated HO-1 knockdown E47 cells confirm the data of cell death. ${ }^{*} \mathrm{p}<0.05$ compared to $3 \mathrm{hr}$ IR treatment; $\# \mathrm{p}<0.01$ compared to $3 \mathrm{hr}$ IR treatment; $\ddagger \mathrm{p}<0.001$ compared to $6 \mathrm{hr}$ IR treatment. 
A.

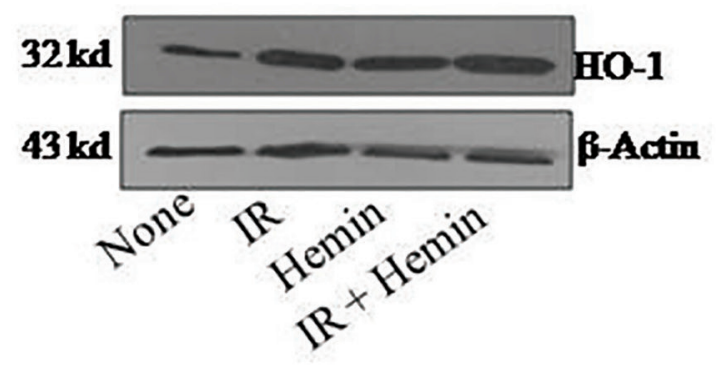

c.

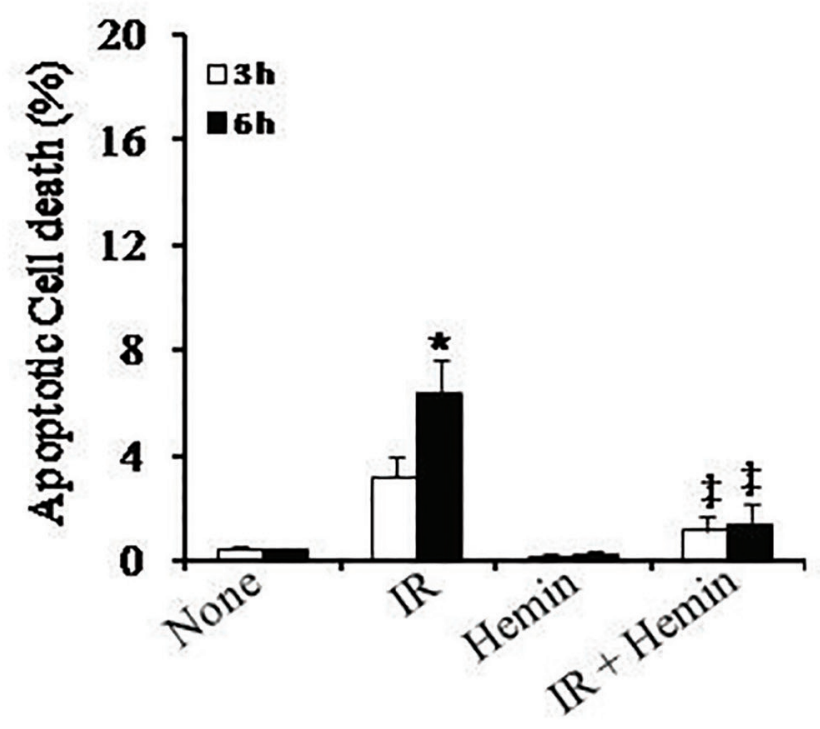

B.

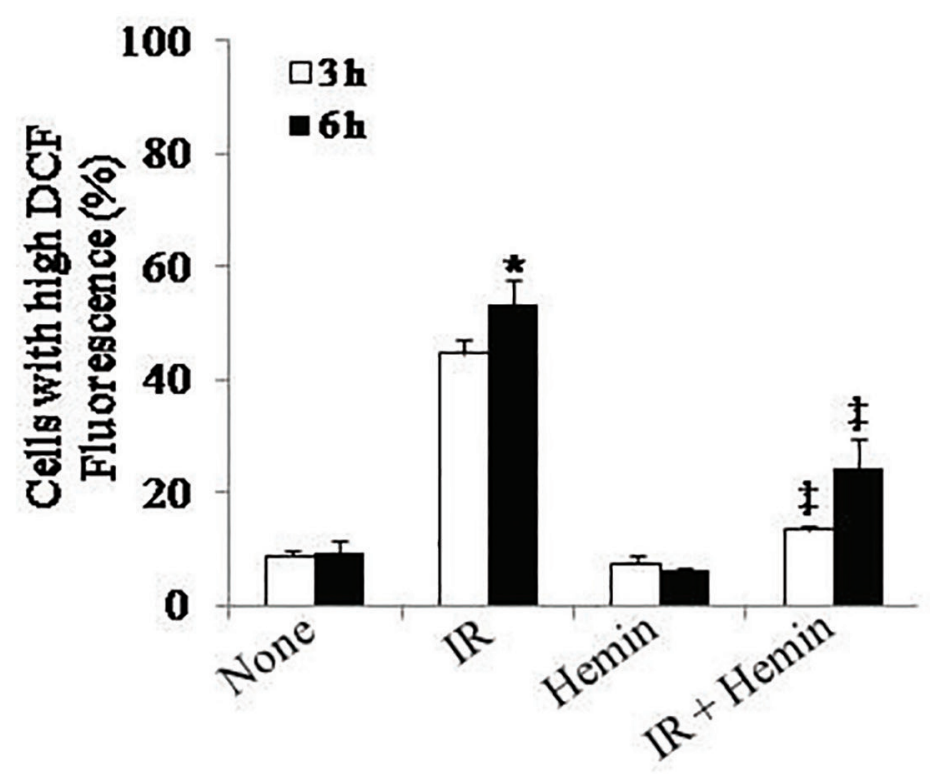

D.

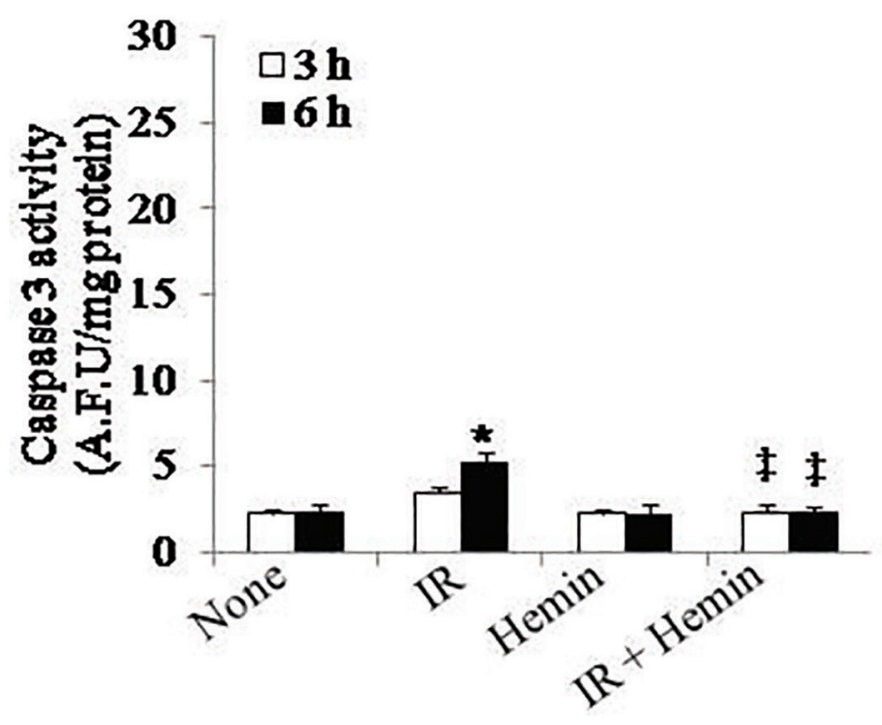

Figure 3: Induction of HO-1 with hemin chloride (hemin) reverses IR induced injury of E47 cells. A) Western blot analysis confirmed the hemin induced increase expression of HO-1 at 6 hrs of IR treatment to E47 cells. B) Reduction of percentage of cells having high DCF fluorescence in E47 cells due to IR treatment for 3 and 6 hrs due to induction of HO-1. C) FACS analysis of propidium iodide and annexin $V$ reveals inhibition of IR induced cell death due to pre-treatment of hemin. D) Pre-treatment of hemin results inhibition of IR induced caspase 3 activity in E47 cells. ${ }^{*} \mathrm{p}<0.05$ compared to $3 \mathrm{hr}$ IR treatment. $\neq \mathrm{p}<0.001$ compared to respective hours of IR treatment alone. 
in loss of protective effect of HO-1 against INH-RMP induced increased oxidative stress and death in E47 cells. Induction of HO-1 by hemin chloride, to investigate the protective role of $\mathrm{HO}-1$, confers resistance of $\mathrm{E} 47$ cells to INH-RMP induced toxicity. HO-1 is also known to be a modulator of cell growth in different cell lines. ${ }^{23}$ Blocking HO-1 by siRNA HO-1 significantly increases the INHRMP induced ROS production and dramatic increase in cell death to E47 cells. HO-1 knockdown enhances the caspase 3 activity in E47 cells after INH-RMP treatment. Activation of HO-1 by INH-RMP serves as an adaptive response to the increased oxidative stress caused by INHRMP in E47 cells.

In conclusion, our findings document that induction of cellular HO-1 increases the resistance of E47 cells to drug induced oxidative injury and it may be a useful therapeutic approach to prevent INH-RMP induced oxidative injury and hepatocyte death.

\section{Acknowledgements:}

The authors are thankfully indebted to Professor Arthur I Cederbaum of Icahn School of Medicine at Mount Sinai, New York, for providing E47 cell line as gift to Centre for Liver Research, IPGME\&R, Kolkata. They acknowledge West Bengal University of Health Science, Kolkata as the study is a part of Ph.D thesis work of Debasree Bishnu. The authors also acknowledge the sincere help of Pratap Pandit and Sudipta Chakraborty for conducting this study.

\section{References:}

1. Kumar R, Bhatia V, Khanal S, Khanal S, Sreenivas V, Datta Gupta $S$ et al. Antituberculosis therapy induced acute liver failure: magnitude, profile, prognosis, and predictors of outcome. Hepatology. 2010; 51:1665-1674.

2. Devarbhavi H, Dierkhising R, Kremers WK. Antituberculosis therapy drug-induced liver injury and acute liver failure. Hepatology. 2010;52:798-799

3. Tostmann A, Boeree MJ, Aarnoutse RE, De Lange W CM, Van Der Ven AJAM, Dekhuijzen R. Antituberculosis drug-induced hepatotoxicity: concise up-to-date review. J Gastroenterol Hepatol. 2008; 23:192-202.

4. Larrey D. Epidemiology and individual susceptibility to adverse drug reactions affecting the liver. Semin Liver Dis. 2002; 22:145-155.

5. Steele MA, Burk RF, DesPrez RM. Toxic hepatitis with isoniazid and rifampin. A meta-analysis. Chest. 1991; 99:465-471.

6. Shibahara S, Mueller RM, Taguchi H. Transcriptional control of rat heme oxygenase by heat shock. J Biol Chem 1987; 262:12889-12892.

7. Ewing JF, Maines MD. Rapid induction of heme oxygenase-1 mRNA and protein by hyperthermia in rat brain: heme oxygenase- 2 is not a heat shock protein. Proc Natl Acad Sci U S A 1991; 88:5364-5368.

8. Applegate LA, Luscher P, Tyrrell RM. Induction of heme oxygenase: a general response to oxidant stress in cultured mammalian cells. Cancer Res 1991; 51:974-978.

9. Takahashi T, Morita K, Akagi R, Sassa S. Heme oxygenase-1: a novel therapeutic target in oxidative tissue injuries. Curr Med Chem 2004; 11:1545-1561.

10. Guo X, Shin VY, Cho CH. Modulation of heme oxygenase in tissue injury and its implication in protection against gastrointestinal diseases. Life Sci 2001; 69:3113-3119.

11. Chen Q and Cederbaum AI. Cytotoxicity and apoptosis produced by cytochrome P4502E1 in HepG2 cells. Mol Pharmacol 1998; 53:638-648.

12. Ding, WX., Ni, H.M., Francesca, D.D., Stolz, D.B., Yin, X.M. Bid-dependent generation of oxygen radicals promotes death receptor activation-induced apoptosis in murine hepatocytes. Hepatology 2004; 40, 403-413.

13. Chowdhury A, Santra A, Bhattacharya K, Ghatak S, Saha RD, Dhali GK. Mitochondrial oxidative stress and permeability transition in Isoniazid and Rifampicin induced liver injury in mice. J Hepatol 2006; 45: 117 - 126

14. Ben-Ari Z, Issan Y, Katz Y, Sultan M, Safran M, Michal LS, Nader GA, Kornowski R, Grief F, Pappo O, and Hochhauser E. Induction of heme oxygenase-1 protects mouse liver from apoptotic ischemia/ reperfusion injury. Apoptosis 2013; 18: 547-55.

15. Immenschuh $S$, Ramadori G. Gene regulation of heme oxygenase-1 as a therapeutic target. Biochem Pharmacol 2000; 60:1121-1128.

16. Lee PJ, Jiang BH, Chin BY, Lyer NV, Alam J, Semenza 
GL, Choi AM. Hypoxia-inducible factor-1 mediates transcriptional activation of the heme oxygenase- 1 in responses to hypoxia. J Biol Chem 1997; 272:5375-5381.

17. Bach FH. Heme oxygenase-1: a therapeutic amplification funnel. FASEB J 2005; 19:1216-1219.

18. Ryan DE, Ramanathan L, Iida S, Thomas PE, Haniu M, Shively JE, Lieber CS, et al. Characterization of a major form of rat hepatic microsomal cytochrome P-450 induced by isoniazid. J Biol Chem 1985; 260:6385-6393.

19. Gong P, Cederbaum AI, Nieto N. Increased expression of cytochrome P4502E1 induces heme oxygenase-1 through ERK MAPK pathway.J. Biol. Chem. 2003; 278: 29 693-700.

20. Dai Y, Cederbaum AI. Cytotoxicity of acetaminophen in human cytochrome P4502E1- transfected HepG2 cells. J. Pharmacol. Exp. Ther. 1995; 273: 1497-505.

21. Huang YS, Chern HD, Su WJ, Wu JC, Chang SC, Chiang $\mathrm{CH}$, et al. Cytochrome P450 2E1 genotype and the susceptibility to antituberculosis drug-induced hepatitis. Hepatology 2003; 37:924-30.

22. Metushi IG, Sanders C; Acute Liver Study Group, Lee WM, Uetrecht J. Detection of anti-isoniazid and anti-cytochrome $\mathrm{P} 450$ antibodies in patients with isoniazid induced liver failure. Hepatology 2014 Mar; 59(3):1084-93.

23. Durante W. Heme oxygenase- 1 in growth control and its clinical application to vascular disease. J Cell Physiol 2003; 195:373-382. 